\title{
Estimating the cost-effectiveness of treatment for prevention of thromboembolic events in at-risk adults with non-valvular atrial fibrillation
}

\author{
Marco Bellone ${ }^{1}$, Lorenzo Pradelli ${ }^{1}$, Mario Bo ${ }^{2}$ \\ ${ }^{1}$ AdRes HE\&OR, Turin, Italy \\ ${ }^{2}$ Geriatria, Città della Salute e della Scienza, Molinette, Turin, Italy
}

\begin{abstract}
INTRODUCTION: The direct oral anticoagulants (DOACs) have demonstrated a more predictable effect and a more favorable risk-benefit ratio compared to the standard oral anticoagulant treatment for the prevention of stroke in patients with non-valvular atrial fibrillation (NVAF).

AIM: To estimate the efficiency of DOACs (apixaban, dabigatran, edoxaban, and rivaroxaban vs. warfarin), in the prevention of clinical events in adult patients with NVAF.

METHODS: A deterministic incremental cost-effectiveness analysis was performed to evaluate the avoidance of a clinical event and the incremental cost per avoided clinical event, in a hypothetical population of 100,000 adult patients with NVAF, over 1-year period. In the absence of head-to-head comparison trials between DOACs, relative risks were derived from a network meta-analysis. Clinical events considered include stroke/systemic embolism (SE) and major bleeding. Only direct health costs related to the management of clinical events and drug acquisition costs were considered. Clinical event management costs were derived from literature and from the Diagnosis Related Group (DRG) tariffs. Net annual treatment costs were calculated based on the daily dose reported in the Summary of Product Characteristics (SPCs) and the ex-factory price of each drug.

RESULTS: Among DOACs, apixaban was associated with the highest net clinical benefit with 1,064 avoided events over 1 year, compared to warfarin (728 major bleeding events and 336 strokes/SE). Furthermore, apixaban is the most efficient DOAC, with a cost per avoided event equal to $€ 16,672$ vs. warfarin $(€ 24,120$ for edoxaban $60 \mathrm{mg}, € 36,777$ for dabigatran $150 \mathrm{mg}$ ).

CONCLUSION: Apixaban has the highest potential net clinical benefit among DOACs for patients with NVAF and the least incremental cost per avoided event for the Italian National Health Service.
\end{abstract}

\section{Keywords}

Direct oral anticoagulants; Thromboembolic events; Non-valvular atrial fibrillation

\section{INTRODUCTION}

Atrial fibrillation (AF) represents the most prevalent form of cardiac arrhythmia. In Europe and the US 1 in 4 middle-aged adults will develop AF and it is estimated that by 2030 in Europe there will be 14-17 million of AF patients with 120,000-215,000 new cases per year [1]. In Italy, a recent observational study estimated a prevalence of AF equal to $1.7 \%$ for a total of 1,036,448 cases [2]. AF is independently associated with a higher risk of all-cause and cardiovascular mortality, the latter due to sudden death, heart failure, and stroke. Compared to subjects without AF, stroke, which is the main complication of AF, is nearly five times more frequent in subjects with $\mathrm{AF}$ [3]. Ischemic stroke due to AF is associated with higher mortality and worse functional outcomes than non AF-associated ischemic stroke, and $\mathrm{AF}$ is responsible for $20-30 \%$ of ischemic stroke [1].

In this context, the prevention of stroke represents a primary goal in the management of patients with AF and a high thromboembolic risk. The current European guidelines recommend to evaluate the annual incidence of cerebral stroke using the CHA2DS-VASc score

Corresponding author Marco Bellone

m.bellone@adreshe.com

Received: 12 January 2018 Accepted: 22 February 2018 


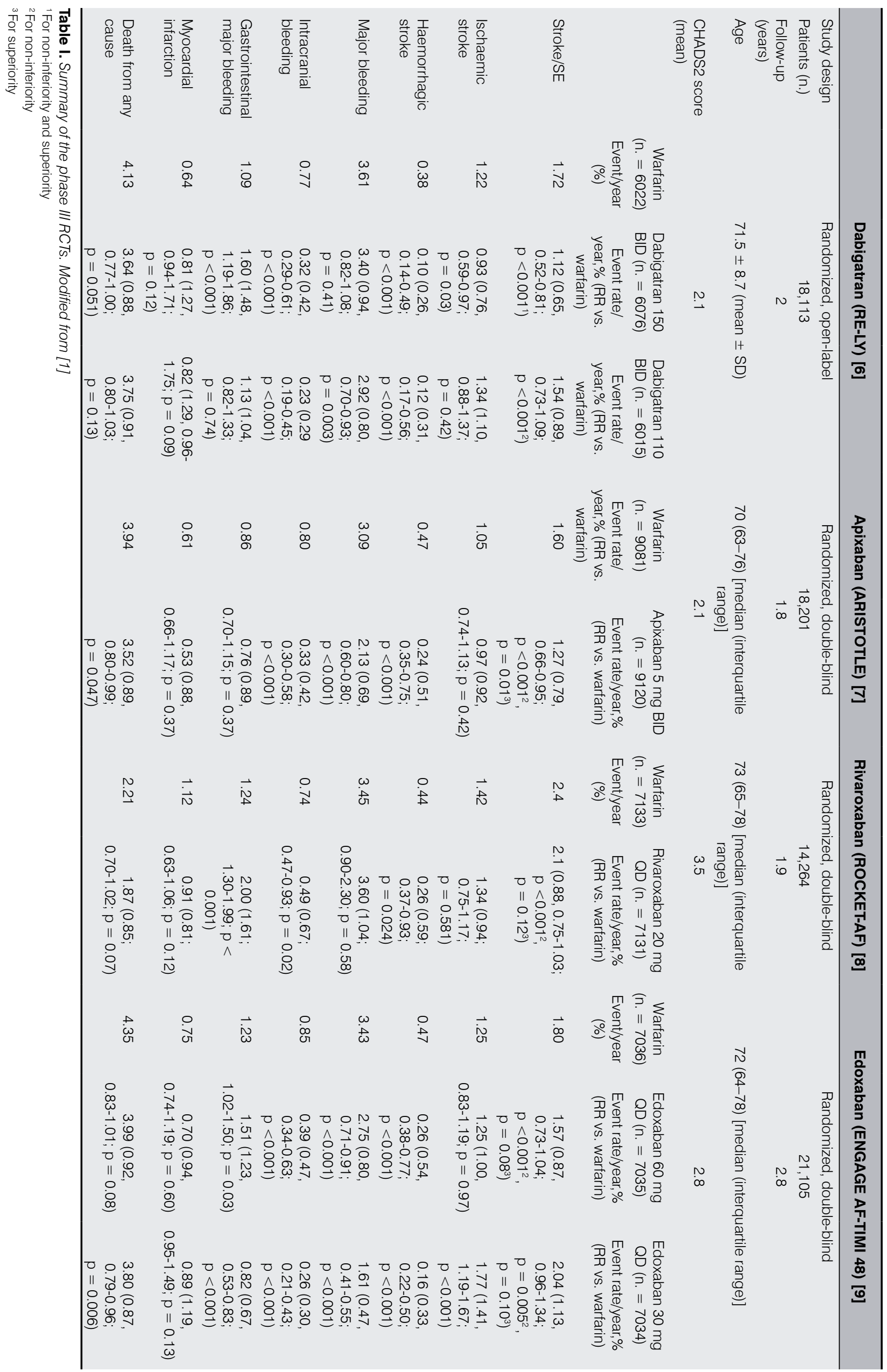


which stratifies patients with thromboembolic risk based on gender (female have a higher risk) and the presence of congestive heart failure, hypertension, age $\geq 65$ years, diabetes, previous stroke or transient ischemic attack (TIA) or thromboembolism, and vascular disease [1]. Oral anticoagulation (OAC) is not indicated in absence of clinical risk factors (CHA2DS2VASc $=0$ - includes women without other stroke risk factors), while is highly recommended in subjects with AF and CHA2DS2-VASC $\geq 2$ (if men) or $\geq 3$ (if women). OAC has potential clinical net benefit also in patients with one risk factor, therefore it should be considered in men with CHA2DS2-VASC $=1$ and women with CHA2DS2-VASC $=2$ [1].

The traditional standard oral anticoagulant treatment is represented by warfarin and other vitamin $\mathrm{K}$ antagonists (VKAs) which have demonstrated to reduce the risk of stroke by $64 \%$ compared with placebo [4]. However, VKA therapy presents some limitations, such as interactions with drugs and food, the need for regular dosage adjustment based on periodic INR (International Normalized Ratio) monitoring, and the risk of major bleeds, which could reduce patient compliance [5]. During the last years new direct anticoagulant drugs which overcome some of these limitations have been developed.

The direct oral anticoagulants (DOACs) have been approved for the prevention of stroke in patients with NVAF (non-valvular AF) thereby excluding AF patients with mechanical heart valves or moderate to severe mitral stenosis. The DOACs currently indicated are factor $\mathrm{Xa}$ inhibitors apixaban, edoxaban, and rivaroxaban; and the direct thrombin inhibitor dabigatran; they have demonstrated a more predictable effect and a more favorable risk-benefit ratio, without the need for INR monitoring, compared to standard therapy. In phase III trials (Table I), and in several indirect comparisons, both the individual DOACs and the entire class were considered non-inferior, or superior, to warfarin in the reduction of stroke, systemic embolism (SE), intracranial bleeding, and mortality [6-14] and are recommended in the first-line treatment of patient with AF in preference to VKAs [1].

When selecting a DOAC for stroke prevention in AF, several factors must be considered, including the evaluation of the net clinical benefit, since the anticoagulant activity, which allows reducing ischemic stroke and systemic embolism, must be balanced against an increased risk of hemorrhagic events.

Despite the overall clinical net benefit of DOACs compared with VKAs, mainly due to reduced incidence of intracranial bleeding, there are important differences in the safety and efficacy profile among DOACs. A recent systematic review and meta-analysis of RCT aimed to identify the most effective, safe, and cost-effective anticoagulant for stroke prevention in $\mathrm{AF}$, demonstrated that apixaban seems to have the best risk benefit ratio $[12,15]$.

The results, obtained from $23 \mathrm{RCTs}$, confirmed that apixaban $5 \mathrm{mg}$ bis in die (BID - twice daily), dabigatran $150 \mathrm{mg}$ BID, edoxaban $60 \mathrm{mg}$ quaque die (QD - once daily), and rivaroxaban $20 \mathrm{mg}$ QD all reduce the risk of stroke or systemic embolism, major bleeding, intracranial bleeding, and all-cause mortality compared with warfarin. Of the available DOACs, apixaban offers the best balance between efficacy and safety since it was ranked the best intervention for many of the outcomes evaluated, including stroke or SE, myocardial infarction, major bleeding, and all-cause mortality [12].

Another network meta-analysis, which included seven trials (for a total of 52,701 patients), showed that, while apixaban $5 \mathrm{mg}$ BID and dabigatran $150 \mathrm{mg}$ BID proved to be equally superior to warfarin in preventing stroke $+\mathrm{SE}$, apixaban was associated with fewer major bleeding events than dabigatran $150 \mathrm{mg}$ BID (OR = 0.73; CI95\%: 0.57-0.93) and rivaroxaban $20 \mathrm{mg}$ $\mathrm{QD}(\mathrm{OR}=0.66$; CI95\%: 0.52-0.84) and fewer drug discontinuations than dabigatran $150 \mathrm{mg}$ $\mathrm{BID}(\mathrm{OR}=0.64$; CI95\%: 0.52-0.78) and $110 \mathrm{mg}$ BID (OR =0.66; CI95\%: 0.54-0. 81). Since the ENGAGE AF-TIMI was ongoing, data for edoxaban were inconclusive [9].

Edoxaban was included in a network meta-analysis of the four phase III RCTs with the aim to assess the relative efficacy and safety of DOACs [11]. The results show that apixaban has a better safety profile in comparison to the other DOACs. Indeed it was associated with fewer bleeding events of any type (OR $=0.81$; CI95\%: 0.89-0.74) than edoxaban $60 \mathrm{mg}$ QD and less major and GI bleeds than dabigatran $150 \mathrm{mg}$ (major: $\mathrm{OR}=0.75$; CI95\%: 0.61-0.90; GI: $\mathrm{OR}=0.59$; CI95\%: 0.41-0.82) and rivaroxaban (major: $\mathrm{OR}=0.67$; CI95\%: 0.82-0.55; GI: OR =0.58; CI95\%: 0.86-0.43) [11]. Finally, an indirect comparison analysis based on the phase III clinical trials found that there were no significant efficacy differences between edoxaban $60 \mathrm{mg}$ QD and apixaban $5 \mathrm{mg}$ BID, but apixaban was associated with lower clinically relevant non-major bleeding $(\mathrm{HR}=0.79$; CI95\%: 0.70-0.90) and gastrointestinal bleeding $(\mathrm{HR}=0.72$; CI95\%: 0.55-0.96) [14]. Regarding gastrointestinal bleeding, the meta-analysis from Ruff et al., which compared DOACs as a class with warfarin, showed that DOACs are associated with significant reductions in stroke, intracranial hemorrhage and mortality, a simi- 
lar risk of major bleeding, and a higher risk of GI bleeding. However, individual comparisons show that, unlike dabigatran $150 \mathrm{mg}$, rivaroxaban and edoxaban $60 \mathrm{mg}$, apixaban is associated with a decreased risk of gastrointestinal bleeding [13].

In a recent consensus document, published on the European Heart Journal $[16,17]$, the Authors give suggestions, based on the results of phase III trials or, if unavailable, on expert opinion, for chosing the drug and/or dose for particular subgroups of patients. In particular, apixaban $5 \mathrm{mg}$ twice daily is recommended as first choice for patients with AF and high risk of gastrointestinal bleeding, chronic kidney failure (creatinine clearance 30-49 $\mathrm{ml} / \mathrm{min}$ ) or older than 75 years .

Aim of this paper is to estimate the efficiency of DOACs (apixaban, dabigatran, edoxaban, and rivaroxaban vs. warfarin) in the prevention of clinical events in adult patients with NVAF from the perspective of the Italian NHS.

\section{METHODS}

A deterministic incremental cost-effectiveness analysis was performed in Microsoft Excel $^{\circledR}$. The effectiveness side is defined as the avoidance of a clinical event, which may be either a stroke/SE (primary efficacy indicator) or a major bleed (primary safety indicator). The overall cost-effectiveness indicator is the incremental cost per avoided clinical event, evaluated in a hypothetical population of 100,000 adult patients with NVAF, with one or more risk factors, such as prior stroke or transient ischaemic attack (TIA); age $\geq 75$ years; hypertension; diabetes mellitus; symptomatic heart failure (NYHA Class $\geq$ II), (Figure 1). Time horizon is one year.

\section{Clinical input}

Clinical events considered in the analysis include the primary efficacy outcome of stroke/ SE and the primary safety outcome of major bleeding. In the absence of RCTs that directly compare apixaban with other DOACs, relative risks were derived from the network metaanalysis (NMA) by Lip et al. [18]. NMA is a type of analysis widely used in pharmacoeconomics for overcoming the lack of head-to-head trials, as reported by the ISPOR Task Force on Indirect Treatment Comparisons Good Research Practices [19] and the NICE guide to the methods of technology appraisal [20].

Lip et al. conducted a network meta-analysis including data from phase III RCTs on full dose apixaban, edoxaban, dabigatran, and rivaroxaban (Table I) vs. warfarin, to assess the relative efficacy and safety in stroke prevention of apixaban vs. other DOACs [18], both on the overall evaluated population and in three subgroups: i) patients with CHADS2 score $\geq 2$; ii) use in secondary prevention (patients with previous stroke or TIA); iii) patients with high quality anticoagulation control with warfarin [18]. The results of the comparison of DOACs with warfarin were consistent with the results from the RCTs and broadly similar in the overall and subgroup results, with apixaban showing the most favorable efficacy and safety profile.

The current analysis is conducted as a series of pairwise comparisons between DOACs and warfarin, using the HRs of the annual risk of stroke/SE and major bleeding (Table II) [18].

In RCTs and NMA analyses, data on

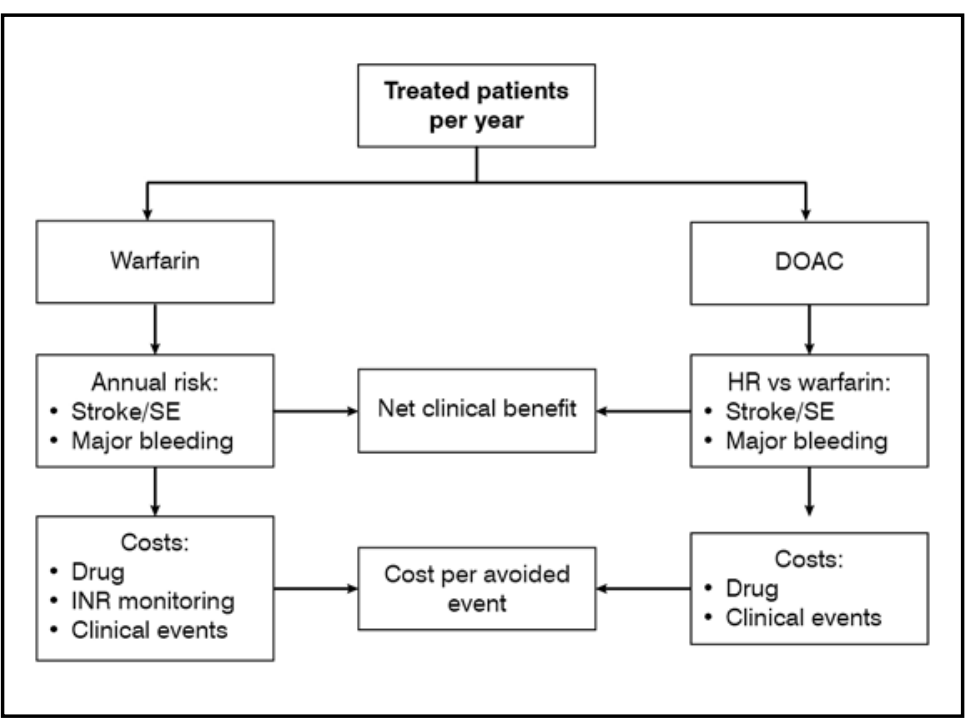

Figure 1. Structure of the cost calculation hemorrhagic strokes are included as part of stroke/SE and major bleeding. In order to avoid double-counting these events in the calculation of the net clinical benefit, the number of hemorrhagic strokes was subtracted from major bleeding events counts (Table II). Similarly, gastrointestinal bleeds, included in major bleeding counts, were not considered as separate clinical events.

\section{Cost input}

Since the analysis was conducted from the perspective of the Italian NHS, only direct health costs related to acquisition and monitoring of drugs and to management of episodes of stroke/SE and major bleeding events were considered. 


\begin{tabular}{lccccc}
\hline & Warfarin Annual & \multicolumn{4}{c}{ HR vs. warfarin (CI95\%) [18] } \\
\cline { 3 - 6 } & Risk (\%) [6] & Apixaban & Dabigatran & Rivaroxaban & Edoxaban \\
& & $\mathbf{5 ~ m g ~ B I D ~}$ & $\mathbf{1 5 0} \mathbf{~ m g ~ B I D ~}$ & 20 mg QD & 60 mg QD \\
\hline Stroke/SE & 1.60 & $0.79(0.66-0.95)$ & $0.65(0.52-0.81)$ & $0.87(0.74-1.03)$ & $0.87(0.75-1.02)$ \\
Major bleeding & 3.09 & $0.69(0.60-0.80)$ & $0.93(0.81-1.07)$ & $1.05(0.91-1.2)$ & $0.80(0.71-0.91)$ \\
Hemorragic stroke & 0.47 & $0.51(0.35-0.74)$ & $0.26(0.13-0.48)$ & $0.59(0.39-0.89)$ & $0.55(0.39-0.78)$ \\
\hline
\end{tabular}

Table II. HR vs. warfarin used in the analyses

\begin{tabular}{lllccccc}
\hline & Daily dose & Package & $\begin{array}{c}\text { Ex-factory } \\
\text { price } \mathbf{(} / \mathbf{d a y})\end{array}$ & $\begin{array}{c}\text { Acquisition } \\
\text { legal price } \\
\text { discount (-5\%) }\end{array}$ & $\begin{array}{c}\text { Legal price } \\
\text { discount } \\
\mathbf{( - 5 \% )}\end{array}$ & $\begin{array}{c}\text { Confidential } \\
\text { discount }\end{array}$ & $\begin{array}{c}\text { Price } \\
\text { volume } \\
\text { agreement }\end{array}$ \\
\hline Apixaban & $5 \mathrm{mg} \mathrm{BID}$ & 60 tablets $5 \mathrm{mg}$ & 2.23 & Yes & $\mathrm{P}$ & Yes & Yes \\
Dabigatran & $150 \mathrm{mg} \mathrm{BID}$ & 60 tablets $150 \mathrm{mg}$ & 2.23 & Yes & $\mathrm{P}$ & Yes & Yes \\
Rivaroxaban & $20 \mathrm{mg} \mathrm{QD}$ & 28 tablets $20 \mathrm{mg}$ & 2.20 & Yes & $\mathrm{P}$ & Yes & No \\
Edoxaban & $60 \mathrm{mg}$ QD & 28 tablets $60 \mathrm{mg}$ & 2.09 & Yes & $\mathrm{A}$ & Yes & No \\
Warfarin & $5 \mathrm{mg} \mathrm{QD}$ & 30 tablets $5 \mathrm{mg}$ & 0.071 & - & - & - & - \\
\hline
\end{tabular}

Table III. Drug acquisition costs (prices refer to 2016)

${ }^{1}$ Retail price

$\mathrm{A}=$ At the time of the acquisition; $\mathrm{P}=$ Returned as payback

\section{Event management costs}

Clinical event management costs over 1 year include all costs incurring during the acute period and any long-term maintenance costs. The annual cost attributed to a major bleed is approximated with the corresponding DRG-based tariff paid to hospitals by the National Health Service [21], in particular DRG 174 - GI major bleed was used ( $€$ 3,317 per event). Stroke/ SE management cost derive from data reported in an observational study conducted on 411 Italian stroke survivors, followed up for 12 months [22]. In this study, the total direct healthcare costs amounted to an average $€ 11,747$ per stroke survivor, considering both ischemic and hemorrhagic stroke.

\section{Drug costs}

Drug acquisition costs were taken from the Italian Official Gazette $[23,24]$ and according with the current legislation apixaban (Eliquis ${ }^{\circledR}$, Pfizer/Bristol-Myers Squibb), dabigatran (Pradaxa ${ }^{\circledR}$, Boehringer Ingelheim), rivaroxaban (Xarelto ${ }^{\circledR}$, Bayer Pharma/Janssen Pharmaceuticals) and edoxaban (Lixiana ${ }^{\circledR}$, Daiichi-Sankyo) are valued using ex-factory prices, while retail price was considered for warfarin (Coumadin ${ }^{\circledR}$, Bristol-Myers Squibb). Net annual treatment costs were calculated based on the daily dose reported in the SPCs of each drug (Table III).

For warfarin treated patients an annual INR monitoring cost of $€ 380$ was considered, as reported by Pradelli et al. [25] in which the annual cost reported by Mennini et al. [26] was actualized to 2013 values.

\section{Sensitivity analysis}

A probabilistic sensitivity analysis (PSA) was performed to take into account uncertainties in the input parameters. This was obtained by a Montecarlo simulation: the simulation was repeated 1,000 times sampling all the key parameters from appropriate distributions fitted on the available mean and standard error (SE). The PSA was conducted on the hazard ratios (HRs) for each event considered (i.e. stroke/SE, major bleeding, hemorrhagic stroke). For the PSA, the natural logarithm was sampled from a normal distribution fitted on the confidence intervals reported. The parameters and the distributions used for the PSA are reported in Appendix A.

\section{RESULTS}

Figure 2 shows the number of stroke/SE and major bleeding events avoided and the net clinical benefit achieved with each DOAC compared to warfarin for the treatment of a hypothetical cohort of 100,000 patients with NVAF, with one or more risk factors over 1-year pe- 


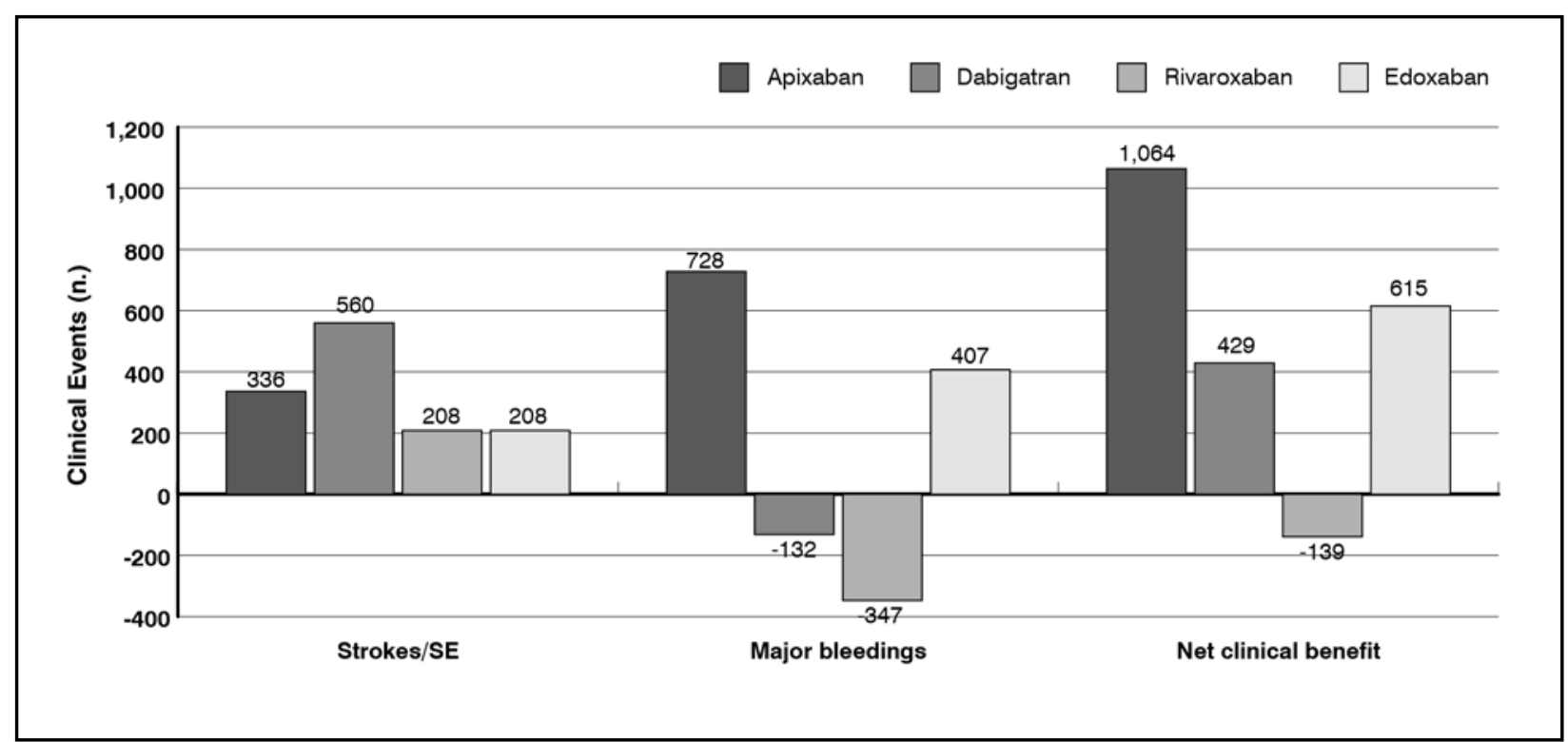

Figure 2. Number of Clinical Events Avoided vs. warfarin (negative values indicate increased number of events with the treatment compared to warfarin)

\begin{tabular}{lccc|cc}
\hline & \multicolumn{3}{c}{ Costs $(\boldsymbol{\epsilon})^{\mathbf{1}}$} & \multicolumn{2}{c}{ Net clinical benefit } \\
\cline { 2 - 6 } & Event-related & Drug & Monitoring & Avoided events (n.) & Cost per avoided event (€) \\
\hline Apixaban vs. warfarin & $-6,360,441$ & $62,092,500$ & $-38,000,000$ & 1,064 & 16,672 \\
Dabigatran vs. warfarin & $-6,142,135$ & $59,535,750$ & $-38,000,000$ & 428 & 35,924 \\
Edoxaban vs. warfarin & $-3,791,737$ & $56,613,750$ & $-38,000,000$ & 615 & 24,120 \\
Rivaroxaban vs. & $-1,291,714$ & $56,613,750$ & $-38,000,000$ & -139 & N/A \\
warfarin & & & & & \\
\hline
\end{tabular}

Table IV. Net costs and net clinical benefit of DOACs vs. warfarin

${ }^{1}$ Negative values indicate savings with the treatment compared to warfarin

riod. Among DOACs, apixaban, with 336 stroke/SE and 728 major bleeding events avoided, showed the highest clinical benefit, followed by edoxaban $60 \mathrm{mg}$, and dabigatran $150 \mathrm{mg}$, while rivaroxaban, according to the methodology used and the outcomes considered, would not seem to have a net clinical benefit, as defined for the purpose of the present analysis, over warfarin.

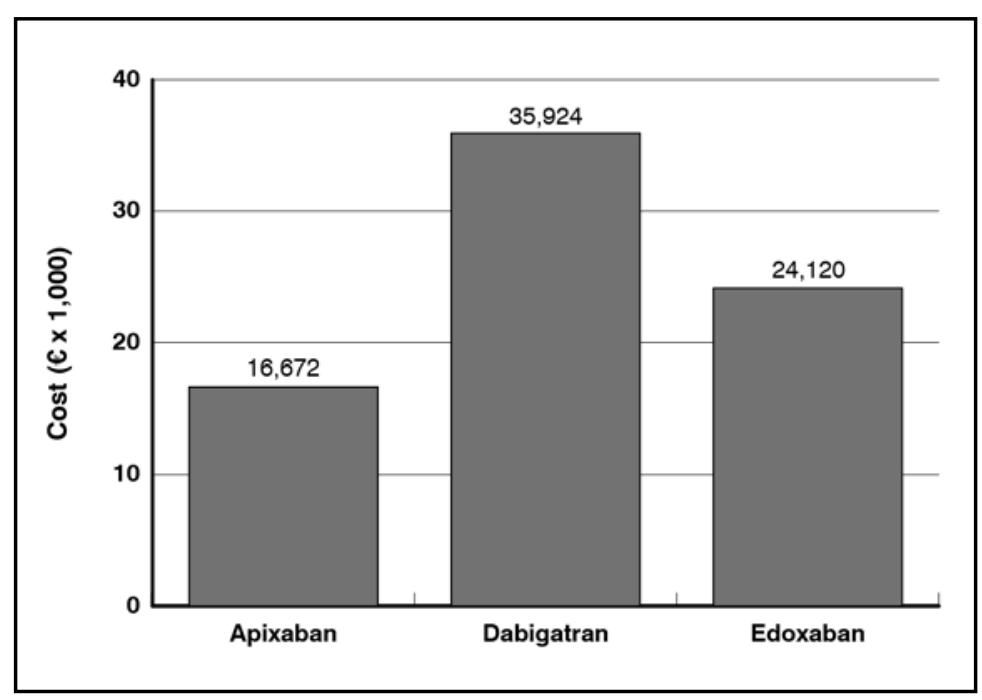

Figure 3. Incremental cost per avoided event (vs. warfarin). The graph does not show the result for rivaroxaban, as its cost per avoided event resulted negative (dominated)
Table IV reports the incremental costs associated with each DOAC vs. warfarin. The higher drug acquisition cost associated with apixaban, compared with other DOACs, is coupled with the lowest incidence of clinical events, resulting in the least cost per avoided event (Figure 3).

\section{Sensitivity analysis}

Figure 4 shows the results of the PSA in terms of cost-effectiveness acceptability curve (CEAC).

\section{DISCUSSION}

In the last years warfarin was progressively substituted by DOACs as anticoagulant therapy for the prevention of stroke in patients with NVAF. In pivotal RCTs, DOACs have demonstrated net clinical benefit compared with warfarin, mainly driven by 


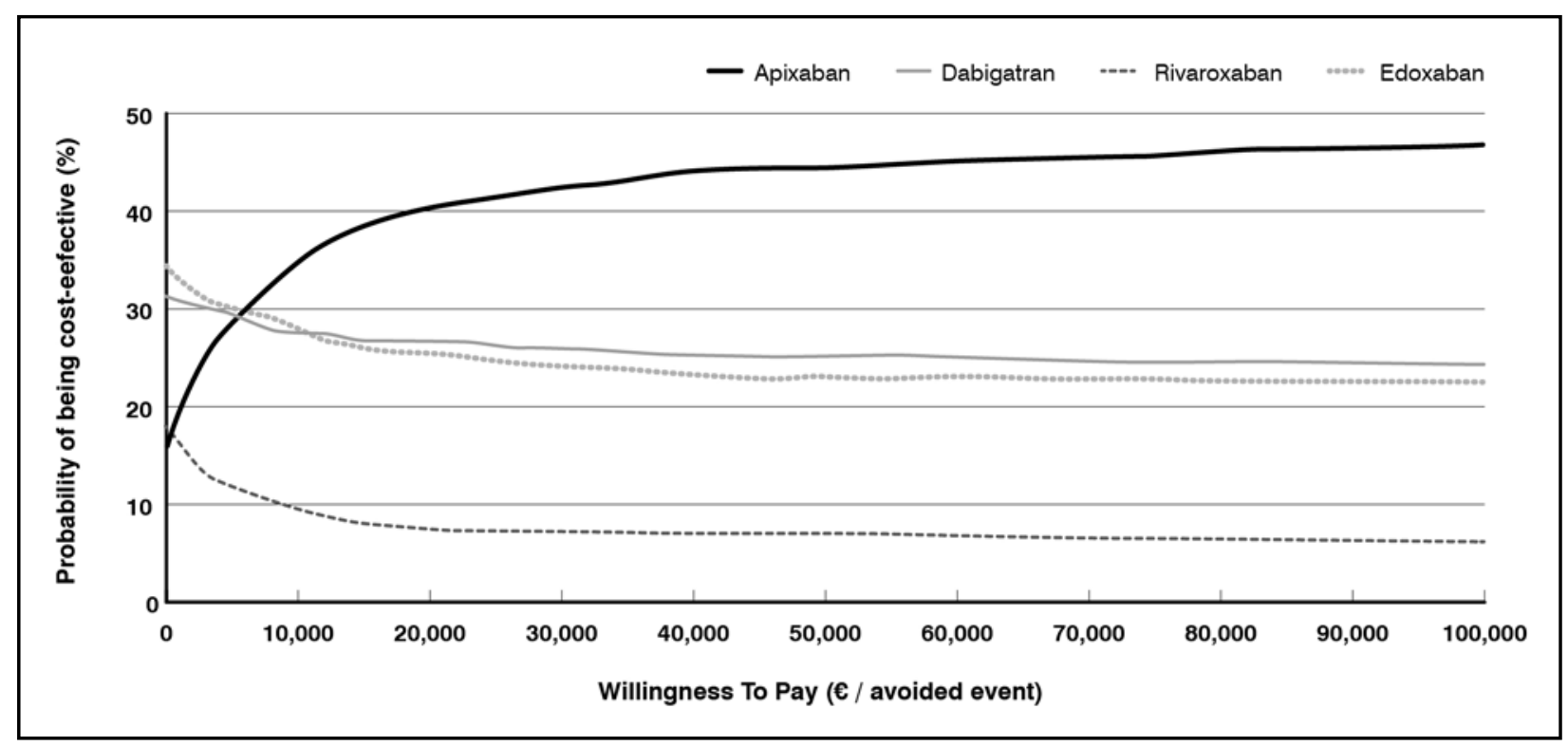

Figure 4. Cost-effectiveness acceptability curve

a significant reduction of intracranial bleedings [6-9]. However, relevant differences in the incidence of major and gastrointestinal bleeding have been observed among DOACs, and apixaban seems to have the best safety profile, with a lower incidence of major bleeding [10$12]$ including gastrointestinal bleeding $[13,14]$.

The economic value of apixaban has been demonstrated in an independent cost-effectiveness analysis conducted in UK and based on the data from a network meta-analysis of 23 RCTs [12], which showed that apixaban has a slightly higher expected quality-adjusted life expectancy (QALYs: 5.49 vs. 5.45 of rivaroxaban, 5.42 of dabigatran $150 \mathrm{mg}$, and 5.41 of edoxaban $60 \mathrm{mg}$ ) and the highest probability of being the most cost-effective first-line therapy for $\mathrm{AF}$ (close to $60 \%$ for willingness to pay thresholds of $£ 20,000-30,000$ ). In a systematic review on the cost-effectiveness of apixaban for stroke prevention, which included 23 costeffectiveness studies from 14 countries, apixaban appears to be more cost-effective than warfarin and other DOACs [27].

In particular, apixaban was cost-effective compared to warfarin according to the costeffectiveness thresholds used in various countries, furthermore all the studies indicated that apixaban was cost-effective, or dominant, with ICERs below the WTP thresholds. The economic evaluations showed a mean ICER equal to $16,502 € /$ QALY $(7,212-57,245 € /$ QALY) and the probability of apixaban to be cost-effective equal to $73.4 \%$.

The evaluation of the net clinical benefit allows balancing efficacy (in terms of reduction of ischemic stroke and systemic embolism) and risk (in terms of hemorrhagic events) of the anticoagulant therapy with DOACs vs. warfarin and, in the absence of direct comparisons, may help clinical decisions.

In the present analysis, which aimed to evaluate the costs associated with the prevention of stroke/SE and major bleeding in adult patients with NVAF, apixaban was associated with 1,064 avoided events over 1 year (728 major bleeding events and 336 strokes/SE), the highest net clinical benefit compared to warfarin among considered DOACs. Furthermore, apixaban was found to be the most efficient DOAC, with the lower incremental cost per avoided event equal to $€ 16,672$ vs. warfarin. Finally, the results of the PSA shown that apixaban had the highest chances to be the most cost-effective treatment for any WTP thresholds higher than $€$ 5,000 per avoided event.

However, equaling the relevance of all clinical events may be a limitation of the methodology we used to estimate the net clinical benefit: as previously pointed out, an approach weighting the number of events by event-specific clinical relevance tariffs may be more accurate in reflecting the global clinical balance among avoided ischemic events and potential excess bleeds [28-31].

A recent Italian research aimed to estimate the net clinical benefit of DOACs vs. warfarin based on data from phase III clinical trials [31] applied this weighing approach to assess the net clinical benefit of the DOACs, as compared to warfarin, in AF patients. The results of this analysis are in agreement with our unadjusted approach in terms of ranking among DOACS, 
with apixaban showing the highest net clinical benefit, followed by edoxaban, dabigatran, and rivaroxaban [31]. However, a notable difference refers to rivaroxaban, that also outperforms warfarin when the net clinical benefit is estimated using the weighed approach. When we repeated our analysis using as input the net clinical benefit reported by Renda et al. [31], our conclusions were unaffected: apixaban is the most efficient DOAC, with a cost per avoided event equal to $€ 13,038$ vs. warfarin ( $€ 14,252$ for edoxaban $60 \mathrm{mg}, € 15,092$ for dabigatran $150 \mathrm{mg}$, and $€ 23,408$ for rivaroxaban).

Another possible limitation of our analysis is the deterministic use of the central estimate of the HRs calculated by Lip et al. [18]; however, the consistence of the results obtained using two different sources as clinical input strengthens our confidence in the main conclusion of the analyses.

A caveat needed in interpreting our results is inherent to the type of comparison feeding the clinical side of the analyses, i.e. indirect comparison - full validity can be claimed for the comparisons of the single DOACs vs. warfarin, in the specific population of AF patients evaluated in the pivotal RCTs, while the comparisons among the former are valid to the extent to which these populations can be regarded as comparable.

Two more limitations to be kept in mind are common to all model-based economic evaluations, and relate to the need to combine different sources in one single conceptual framework, and to the limited transferability of the economic result from one setting to others - these results are Italy specific.

\section{CONCLUSIONS}

Several studies and meta-analyses suggest that apixaban has the highest potential net clinical benefit among DOACs for patients with NVAF. Our findings demonstrate that the higher drug acquisition cost associated with apixaban is coupled with the lowest incidence of clinical events, resulting in the least incremental cost per avoided event from the perspective of the Italian health service.

\section{Funding}

The conduct of the analysis and the publication of this article were sponsored by Pfizer Italy and Bristol-Myers Squibb Italy. Medical writing support and journal styling services were provided by SEEd Medical Publishers and funded by Pfizer Italy and Bristol-Myers Squibb Italy.

\section{Conflict of interest}

M. Bellone is employee of AdRes, which has received project funding by Pfizer Italy and Bristol-Myers Squibb Italy for the conduct of the analysis.

LP is co-owner and employee of AdRes, which has received project funding by Pfizer Italy and Bristol-Myers Squibb Italy for the conduct of the analysis. LP is an editorial member of Farmeconomia. Health Economics and Therapeutic Pathways.

M. Bo has received honoraria from Pfizer Italy and Bristol-Myers Squibb Italy, Boehringer Italy, and Bayer Italy for scientific collaborations.

\section{REFERENCES}

1. Kirchhof P, Benussi S, Kotecha D, et al. 2016 ESC Guidelines for the management of atrial fibrillation developed in collaboration with EACTS. Eur Heart J2016; 37: 2893-962; https:// doi.org/10.1093/eurheartj/ehw210

2. Andreotti F, D’Angela D, Mancusi RL, et al. Prevalence of atrial fibrillation, treatment eligibility and consumption of oral anticoagulants in Italian Local Health Authorities: impact of non-vitamin K antagonist oral anticoagulants. G Ital Cardiol 2017; 18: 230-38; https:// doi.org/10.1714/2674.27400

3. Wolf PA, Abbott RD, Kannel WB. Atrial fibrillation as an independent risk factor for stroke: the Framingham Study. Stroke 1991; 22: 983-8; http://dx.doi.org/10.1161/01.STR.22.8.983

4. Hart RG, Pearce LA, Aguilar MI. Meta-analysis: antithrombotic therapy to prevent stroke in patients who have nonvalvular atrial fibrillation. Ann Intern Med 2007; 146: 857-67; http://dx.doi.org/10.7326/0003-4819-146-12-200706190-00007

5. Ansell J, Hirsh J, Hylek E, et al.; American College of Chest Physicians. Pharmacology and management of the vitamin K antagonists: American College of Chest Physicians Evidence- 
Based Clinical Practice Guidelines (8th Edition). Chest 2008; 133(6 Suppl): 160S-198S; https://doi.org/10.1378/chest.08-0670

6. Connolly SJ, Ezekowitz MD, Yusuf S, et al.; RE-LY Steering Committee and Investigators. Dabigatran versus warfarin in patients with atrial fibrillation. $N$ Engl J Med 2009; 361: 1139-51; http://dx.doi.org/10.1056/NEJMoa0905561

7. Granger CB, Alexander JH, McMurray JJ, et al.; ARISTOTLE Committees and Investigators. Apixaban versus warfarin in patients with atrial fibrillation. $N$ Engl J Med 2011; 365: 981-92; http://dx.doi.org/10.1056/NEJMoa1107039

8. Patel MR, Mahaffey KW, Garg J, et al.; ROCKET AF Investigators. Rivaroxaban versus warfarin in nonvalvular atrial fibrillation. $N$ Engl J Med 2011; 365: 883-91; http://dx.doi. org/10.1056/NEJMoa1009638

9. Giugliano RP, Ruff CT, Braunwald E, et al.; ENGAGE AF-TIMI 48 Investigators. Edoxaban versus warfarin in patients with atrial fibrillation. N Engl J Med 2013; 369: 2093-104; http://dx.doi.org/10.1056/NEJMoa1310907

10. Biondi-Zoccai G, Malavasi V, D’Ascenzo F, et al. Comparative effectiveness of novel oral anticoagulants for atrial fibrillation: evidence from pair-wise and warfarin-controlled network meta-analyses. HSR Proc Intensive Care Cardiovasc Anesth 2013; 5: 40-54

11. Fu W, Guo H, Guo J, et al. Relative efficacy and safety of direct oral anticoagulants in patients with atrial fibrillation by network meta-analysis. J Cardiovasc Med (Hagerstown) 2014; 15: 873-9; http://dx.doi.org/10.2459/JCM.0000000000000206

12. Sterne JA, Bodalia PN, Bryden PA, et al. Oral anticoagulants for primary prevention, treatment and secondary prevention of venous thromboembolic disease, and for prevention of stroke in atrial fibrillation: systematic review, network meta-analysis and cost-effectiveness analysis. Health Technol Assess 2017; 21: 1-386; http://dx.doi.org/10.3310/hta21090

13. Ruff CT, Giugliano RP, Braunwald E, et al. Comparison of the efficacy and safety of new oral anticoagulants with warfarin in patients with atrial fibrillation: a meta-analysis of randomised trials. Lancet 2014; 383: 955-62; http://dx.doi.org/10.1016/S0140-6736(13)62343-0

14. Skjøth F, Larsen TB, Rasmussen LH, et al. Efficacy and safety of edoxaban in comparison with dabigatran, rivaroxaban and apixaban for stroke prevention in atrial fibrillation. An indirect comparison analysis. Thromb Haemost 2014; 111: 981-8; http://dx.doi.org/10.1160/ TH14-02-0118

15. López-López JA, Sterne JAC, Thom HHZ, et al. Oral anticoagulants for prevention of stroke in atrial fibrillation: systematic review, network meta-analysis, and cost effectiveness analysis. $B M J$ 2017; 359: j5058

16. Diener HC, Aisenberg J, Ansell J, et al. Choosing a particular oral anticoagulant and dose for stroke prevention in individual patients with non-valvular atrial fibrillation: part 1. Eur Heart J 2017; 38: 852-859; http://dx.doi.org/10.1093/eurheartj/ehv643

17. Diener HC, Aisenberg J, Ansell J, et al. Choosing a particular oral anticoagulant and dose for stroke prevention in individual patients with non-valvular atrial fibrillation: part 2. Eur Heart J 2017; 38: 860-8; http://dx.doi.org/10.1093/eurheartj/ehw069

18. Lip GY, Mitchell SA, Liu X, et al. Relative efficacy and safety of non-Vitamin K oral anticoagulants for non-valvular atrial fibrillation: Network meta-analysis comparing apixaban, dabigatran, rivaroxaban and edoxaban in three patient subgroups. Int J Cardiol 2016; 204: 88-94; http://dx.doi.org/10.1016/j.ijcard.2015.11.084

19. Jansen JP, Fleurence R, Devine B, et al. Interpreting indirect treatment comparisons and network meta-analysis for health-care decision making: report of the ISPOR Task Force on Indirect Treatment Comparisons Good Research Practices: part 1. Value Health 2011; 14: 417-28; https://doi.org/10.1016/j.jval.2011.04.002

20. NICE. Guide to the methods of technology appraisal 2013. Process Published: 4 April 2013 https://www.nice.org.uk/process/pmg9/resources/guide-to-the-methods-of-technologyappraisal-2013-pdf-2007975843781

21. Tariffe delle prestazioni di assistenza ospedaliera per acuti (sistema DRG). Supplemento ordinario n. 8 alla Gazzetta Ufficiale Serie generale - n. 23 del 28-1-2013 
22. Fattore G, Torbica A, Susi A, et al. The social and economic burden of stroke survivors in Italy: a prospective, incidence-based, multi-centre cost of illness study. BMC Neurology 2012; 12: 137; http://dx.doi.org/10.1186/1471-2377-12-137

23. OG General Series No. 246 of 22-10-2014. Available at http://www.gazzettaufficiale.it/eli/ $\mathrm{gu} / 2014 / 10 / 22 / 246 / \mathrm{sg} / \mathrm{pdf}$

24. OG General Series No. 198 of 25-08-2016. Available at http://www.gazzettaufficiale.it/eli/ $\mathrm{gu} / 2016 / 08 / 25 / 198 / \mathrm{sg} / \mathrm{pdf}$

25. Pradelli L, Calandriello M, Di Virgilio R, et al. Comparative pharmacoeconomic assessment of apixaban vs. standard of care for the prevention of stroke in Italian atrial fibrillation patients. Farmeconomia. Health economics and therapeutic pathways 2014; 15(Suppl 1): 15-25; http://dx.doi.org/10.7175/fe.v15i1S.974

26. Mennini FS, Russo S, Marcellusi A. Budget impact analysis resulting from the use of dabigatran etexilate in preventing stroke in patients with non-valvular atrial fibrillation in Italy. Farmeconomia. Health economics and therapeutic pathways 2012; 13: 121-31; http:// dx.doi.org/10.7175/fe.v13i3.268

27. Pinyol C, Cepeda JM, Roldan I, et al. A Systematic literature Review on the Cost-Effectiveness of Apixaban for Stroke Prevention in Non-valvular Atrial Fibrillation. Cardiol Ther 2016; 5: 171-186

28. Connolly S, Pogue J, Hart R, et al. Clopidogrel plus aspirin versus oral anticoagulation for atrial fibrillation in the Atrial fibrillation Clopidogrel Trial with Irbesartan for prevention of Vascular Events (ACTIVE W): a randomised controlled trial. Lancet 2006; 367: 1903-12

29. Connolly SJ, Pogue J, Hart RG, et al. Effect of clopidogrel added to aspirin in patients with atrial fibrillation. $N$ Engl J Med 2009a; 360: 2066-78

30. Eikelboom JW, Connolly SJ, Hart RG, et al. Balancing the benefits and risks of 2 doses of dabigatran compared with warfarin in atrial fibrillation. $J$ Am Coll Cardiol 2013; 62: 900-8

31. Renda G, di Nicola M, De Caterina R. Net Clinical Benefit of Non-vitamin K Antagonist Oral Anticoagulants Versus Warfarin in Phase III Atrial Fibrillation Trials. Am J Med 2015; 128: 1007-14; http://dx.doi.org/10.1016/j.amjmed.2015.03.034

\section{APPENDIX A}

\begin{tabular}{lcccc}
\hline \multicolumn{1}{c}{ Event } & $\begin{array}{c}\text { Expected } \\
\text { value }{ }^{1}\end{array}$ & $\begin{array}{c}\text { Distribution } \\
\text { type }\end{array}$ & Mean & SE $^{2}$ \\
\hline Apixaban vs. warfarin & $-0,236$ & Normal & $-0,236$ & 0,357 \\
Stroke/SE & $-0,371$ & Normal & $-0,371$ & 0,282 \\
Major bleeding & $-0,673$ & Normal & $-0,673$ & 0,734 \\
Hemorragic stroke & & Normal & $-0,431$ & 0,434 \\
Dabigatran vs. warfarin & $-0,431$ & Normal & $-0,073$ & 0,273 \\
Stroke/SE & $-0,073$ & Normal & $-1,347$ & 1,280 \\
Major bleeding & $-1,347$ & & & \\
Hemorragic stroke & & Normal & $-0,139$ & 0,324 \\
Rivaroxaban vs. warfarin & $-0,139$ & Normal & 0,049 & 0,271 \\
Stroke/SE & 0,049 & Normal & $-0,528$ & 0,809 \\
Major bleeding & $-0,528$ & & & \\
Hemorragic stroke & & Normal & $-0,139$ & 0,301 \\
Edoxaban vs. warfarin & $-0,139$ & Normal & $-0,223$ & 0,243 \\
Stroke/SE & $-0,223$ & Normal & $-0,598$ & 0,679 \\
\hline Major bleeding & $-0,598$ & &
\end{tabular}

Table IA. Parameters and distributions used for the PSA

${ }^{1}$ These values represent the natural logarithm of reported HRs

${ }^{2}$ Obtained from reported $\mathrm{C} 195 \%$ 